\section{Let's Focus on Value}

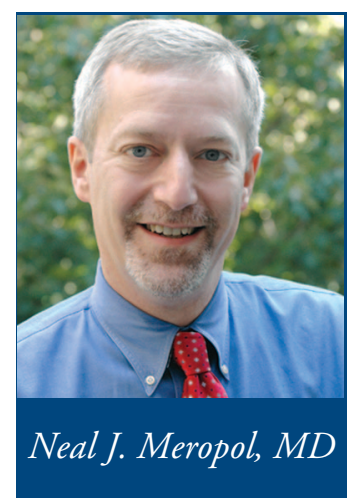

\section{By Neal J. Meropol, MD}

Cancer diagnosis and treatment is expensive, and the price tag is increasing at a rapid rate. However, the cost of cancer care is a small percentage of overall health care expenditures in the United States, and oncologists are unlikely to single-handedly bankrupt the economy. Why then, is there so much angst over the cost of cancer care? After all, is not society's willingness to pay for new health care innovation merely a reflection of our insatiable thirst for (and a driver of) such innovation in a market-based economy?

In this issue of Journal of Oncology Practice, Maurie Markman $^{1}$ expresses the concern that price may negatively impact access, and that payors may make coverage decisions driven by fiscal rather than scientific grounds. He then argues that pricing of cancer treatment should be based on a "rigorously objective and openly debated scientific process that will determine the evidence of the utility of that therapy." These comments underscore an increasingly common experience for the cancer patients we serve, namely the impact of the cost of treatment on patients and their families. It is not only the uninsured, but also those with insurance who must integrate the threat of out-of-pocket expenses (ie, high deductibles, coinsurance, and copayments) into their treatment decisions. Although one might argue that the market drivers that ultimately define the nature and extent of coverage provided by a private or public insurer are the employees and tax payers who purchase the insurance, the bureaucratic complexity of the current systems does not facilitate transparency, advocacy, or a consistent approach to decision making.

I do not view willingness to pay as a conflict between fiscal and scientific considerations. Rather, the willingness to pay

\section{Reference}

1. Markman M: Refocusing the debate: Evidence-based clinical cancer research versus marketplace reality. J Oncol Pract 4:262, 2008 for a particular intervention (whether the decision maker is a patient or payor) represents an integration of these interests. In economic terms, willingness to pay defines value. Thus, value is not an intrinsic characteristic of a new medical intervention, it is an expression of what we think it is worth. A key consideration in assessing value is the overall benefit of the treatment. Insofar as benefit is defined by well-conducted clinical research, value is thus an integration of scientific and fiscal measures. In contrast to many other countries, in the United States we have shied away from discussion of value. Why? Perhaps it is because such discussion would require explicit consideration of cost and effectiveness, leading to difficult decisions regarding thresholds for payment and ultimately explicit rationing of care. Furthermore, conflict may arise because various stakeholders weigh the potential benefits of a particular intervention differently.

I believe that the continued development and licensing of modestly beneficial high-priced treatments, and the potential for widening disparities in cancer care, together warrant a renewed focus on the value of new innovation in cancer care. This focus requires consensus regarding how we measure and define benefits and costs. We must hear and integrate the perspectives of all stakeholders, including patients, providers, producers of new technology, and payors. The goals are shared: continued improvement and access to high-quality, affordable health care for all citizens. The question is whether we as a society are prepared to both accept that resources are finite, and act on the premise that not all innovations are of equal value.

Neal J. Meropol, MD, is Director, Gastrointestinal Cancer Program, at Fox Chase Cancer Center; Senior Fellow, the Center for Bioethics and Adjunct Senior Fellow at the Leonard Davis Institute of Health Economics at the University of Pennsylvania in Philadelphia, PA

DOI: 10.1200/JOP.0851502 